\title{
INVESTIGACIÓN, CREATIVIDAD Y APRENDIZAJE EN LA EDUCACIÓN SUPERIOR
}

\author{
Hernán Zuluaga Albarracín y Juan Carlos Manrique Mosquera \\ Semillero Siembra \\ Grupo de Investigación en Pedagogía \\ Institución Universitaria Antonio José Camacho \\ Recepción: 30/07/2019. Aceptado: 03/12/2019. \\ Cómo citar este artículo: \\ Zuluaga Albarracín, H, y Manrique Mosquera, J.C. (2020). Investigación, creatividad y aprendizaje en la educación \\ superior. Revista Sapientía, 12 (23), 3-12
}

\section{RESUMEN}

En el presente artículo se realiza una reflexión en torno al campo que delimita la intersección de los conceptos creatividad, investigación y aprendizaje en la Educación Superior y cómo desde las diferentes perspectivas de trabajo de cada uno de ellos es posible establecer una relación con los otros.

Esta relación se desarrolla desde los diferentes tipos de trabajo de grado, profundizando en la sistematización de experiencias como una alternativa que permite reflexionar en torno al impacto de la investigación aplicada en las comunidades sociales y académicas.

Son dos las tesis centrales que se desarrollan:

1. La investigación es un campo de trabajo que requiere y propicia un pensamiento creativo, permite realizar procesos de aprendizaje y alcanzar los objetivos de la educación superior.

2. El diseño metodológico es un punto crucial en el proceso investigativo que pone en evidencia la creatividad del investigador como tal o del investigador en formación, para resolver las preguntas que orientan su trabajo.

Esta reflexión es producto de las discusiones académicas que se dan al interior del Grupo de Investigación en Pedagogía (GIP) (categoría C, Colciencias), y del Semillero de Investigación Siembra, de la Facultad de Educación a Distancia y Virtual de la Institución Universitaria Antonio José Camacho.

\section{PALABRAS CLAVES}

Aprendizaje, Educación Superior, Creatividad e Investigación. 


\section{ABSTRACT}

In this article a reflection is made around the field that delimits the intersection of creativity, research and learning in Higher Education and how from the different work perspectives of each of them it is possible to establish a relationship with the others.

This relationship develops from different types of degree work, deepening the systematization of experiences as an alternative that allows us to reflect on the impact of applied research in social and academic communities.

There are two central theses that are developed:

1. Research is a field of work that requires and encourages creative thinking, allows learning processes and achieve the goals of higher education.
2. The methodological design is a crucial point in the research process that highlights the creativity of the researcher as such or of the researcher in training, to solve the questions that guide their work.

This reflection is the product of the academic discussions that take place within the Pedagogy Research Group (GIP) (category C, Colciencias), and the Seed Research Seedbed, of the Faculty of Distance and Virtual Education of the Antonio University Institution José Camacho.

\section{KEYWORDS}

Learning, Higher Education, Creativity and Research.

\section{INTRODUCCIÓN}

El artículo responde a la articulación de dos proyectos de investigación aprobados por el Decanato de Investigaciones. El primero ${ }^{1}$, promueve una reflexión en torno al lugar y la función que tienen los procesos de investigación, los profesores que orientan cursos de investigación y los que dirigen trabajos de grado, para la formación y cualificación profesional de los estudiantes; el segundo ${ }^{2}$, que aborda la cualificación investigativa como parte de las estrategias de formación profesional en atención educativa a la diversidad, desde los procesos de investigación asociados a ese objeto de estudio.

1 "Estrategia pedagógica para el mejoramiento de las competencias investigativas para la dirección de los trabajos de grado de los estudiantes de la licenciaturas en educación de la UNIAJC", del Doctorado en Ciencias Pedagógicas de la Universidad de Ciencias Pedagógicas Enrique José Varona - La Habana - Cuba. La investigación está avalada por la Decanatura Asociada de Investigaciones (PD 0618), y se articula en la sub-línea Gerencia de Procesos Educativos, área de Formación Académica del Grupo de Investigación en Pedagogía (GIP) del Programa de Pedagogía Infantil de la Institución Universitaria Antonio José Camacho (UNIAJC).

2 "Estrategia Pedagógica para la Formación del Profesional en Atención Educativa a la Diversidad en la Licenciatura en Pedagogía Infantil de la UNIAJC" del Doctorado en Ciencias Pedagógicas de la Universidad de Ciencias Pedagógicas Enrique José Varona - La Habana - Cuba. La investigación está avalada por la Decanatura Asociada de Investigaciones y se articula en la sub-línea Inclusión y Diversidad de la línea de investigación Educación y Cultura del Grupo de Investigación en Pedagogía (GIP) del Programa de Pedagogía Infantil de la Institución Universitaria Antonio José Camacho (UNIAJC). 
Se parte de los conceptos: Aprendizaje, Educación Superior, Creatividad e Investigación y del tipo de fenómenos o realidades que involucran, para de esta manera, establecer líneas de argumentación que permiten ver la convergencia a partir de los trabajos de investigación.

Se destaca que los procesos de la investigación son procesos que involucran un componente de creatividad y no son el seguimiento de una serie de pasos o apartes de un documento recetario, con el que se cumplen los requerimientos de una investigación; se pone de relieve, ante todo, el rol activo del investigador frente al fenómeno que indaga desde su reconocimiento, su caracterización o manipulación.

\section{DESARROLLO}

El primer concepto a clarificar es el de aprendizaje como una cualidad intrínseca del ser humano para después vincularlo al sistema de educación; luego, se puede entender como un proceso que se da de manera natural a lo largo de la vida de cualquier persona.

Es así como, en el transcurso de la existencia humana se tienen experiencias directas e indirectas que enriquecen el conocimiento de la realidad y, a partir de ellas, se opera en los contextos en que se vive, aquí no existe una intencionalidad clara o explicita de las personas del entorno por alcanzar ciertos objetivos de aprendizaje.

Cuando la intención de que se aprenda algo existe, se habla de procesos de aprendizaje mediados por el otro y se remite a procesos de enseñanza, los cuales tienden a darse en contextos formales e informales que median el tipo de relación que se establece entre las personas que intervienen en los procesos de enseñanza - aprendizaje (Manrique Mosquera \& Zuluaga Albarracín, 2015).

La educación superior se constituye en un espacio privilegiado para la formación de personas a nivel, técnico, tecnológico, profesional y de posgrado, capaces de dar respuestas a las necesidades de la socie- dad, esta postura implica un reconocimiento de los aspectos involucrados en un determinado problema, el tipo de relaciones que se establecen entre ellos y en términos prospectivos la manera de incidir en los problemas (Manrique Mosquera \& Zuluaga Albarracín, 2015).

Estos aspectos de la educación superior se relacionan, en gran medida, con un paradigma de orden investigativo, es decir, la estructura de los procesos de investigación permite el logro de los objetivos de la educación superior, pero cuando se asume como un proceso orientado a mejorar la calidad de vida desde la solución de problemas y no como la transmisión de conocimientos y saberes.

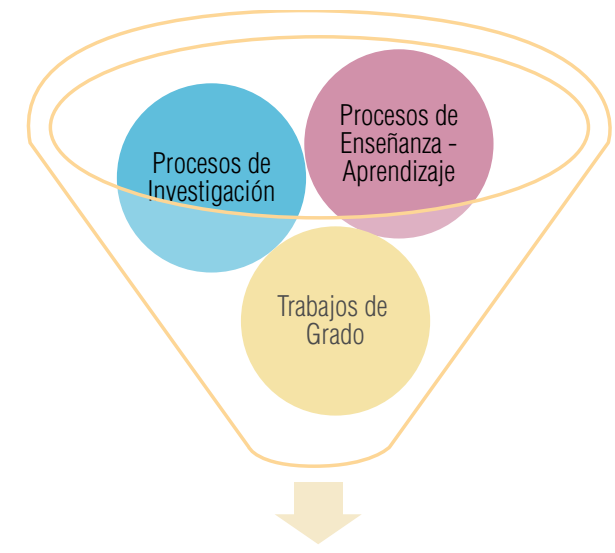

\section{COMPETENCIAS INVESTIGATIVAS}

Gráfica 1. Objetivos de la educación superior

La relación entre creatividad e investigación se puede establecer desde dos perspectivas:

1. La creatividad como objeto de investigación

2. La creatividad como aspecto inherente al proceso investigativo.

En la primera perspectiva, la creatividad como objeto de investigación, es abordada para trata de comprender y definir su naturaleza y los aspectos asociados a su desarrollo. Esta mirada pretende conocer el fenómeno para establecer acciones tendientes a su control, incidiendo en su producción 
o desarrollo y asociándola principalmente al emprendimiento y la resolución de problemas.

Al respecto (Huidobro Salas \& Gonzáles Marquéz, 2005), en su tesis de maestría titulada "Una definición de la creatividad a través del estudio de 24 autores seleccionados" establece cuatro aspectos en torno a los que es posible agrupar las diversas definiciones:

1. La "guestalt" o de la "percepción", donde el énfasis principal se dirige a la recombinación de ideas o la reestructuración de una "guestalt".

2. Las orientadas al producto final o a la innovación.

3. Las de orden "psicoanalítico" o "dinámico"; en las que se señala reestructuración interna del individuo.

4. Las de "énfasis en el proceso", en las que se enfatiza en los procesos del pensamiento más que en la solución del problema.

Desde la segunda perspectiva, la creatividad como un aspecto inherente a los procesos investigativos $y$, por extensión, a los procesos de aprendizaje en la educación superior, realizaremos un análisis de la clasificación anterior para mostrar cómo estos aspectos constitutivos y diferenciales de las definiciones de creatividad se encuentran presentes en los procesos investigativos; tenemos entonces que:

La definición desde la "guestalt" o de la "percepción", se presenta cuando el investigador establece la relación entre los fenómenos objeto de su trabajo, los objetivos, el marco teórico, la metodología, junto con los productos de la investigación, su análisis, y discusión, etc. como un todo que obliga a realizar diferentes ajustes en cada uno de los componentes y momentos de la investigación. El investigador está atento a los elementos constitutivos de la realidad que pretende conocer o intervenir, cómo interactúan los elementos entre sí y como se afectan en el proceso investigativo, para realizar las transformaciones que le permitan aprehender el fenómeno.

Las definiciones orientadas al "producto final o a la innovación", son inherentes al proceso investigativo desde las nuevas maneras de concepción del fenómeno, un fenómeno adquiere otro matiz cuando se inicia una nueva investigación. Con quién o dónde se realiza, la manera como se aborda el problema desde la recolección de la información hasta el análisis de los datos y su posterior discusión implican innovación, es decir, una manera distinta de concebir o abordar el fenómeno objeto de estudio con la intención de generar nuevo conocimiento.

El tercer tipo de definiciones, las de orden "psicoanalítico" o "dinámico"; permiten realizar unas reflexiones desde las características del investigador y el tipo de impulsos que orientan su quehacer, y cómo los fenómenos que indagan lo movilizan y lo llevan a asumir nuevas posturas frente a la realidad indagada; en este sentido, existe una reestructuración interna del investigador en virtud de las transformaciones que se generan al conocer el fenómeno indagado.

Por último, las definiciones con "énfasis en el proceso", que se centran en el proceso de pensamiento, la observamos en la perspectiva que asume el investigador en torno a la información que arroja su investigación, sea esta de carácter teórico y/o práctico, ajustando su postura personal frente a los datos e información de la investigación y que lo llevan a concebir nuevas maneras de pensar y tratar su objeto de investigación.

Ampliando la idea anterior, Guilford (1959) citado por Huidobro Salas \& Gonzáles Marquéz (2005), define la creatividad tomando en cuenta numerosos factores intelectuales, entre ellos, los más importantes son los de descubrimiento y los de pensamiento divergente. Los primeros están definidos como los que tienen la capacidad para desarrollar información de lo que viene dado en forma de estimulación. Los segundos, es decir, los factores de 
pensamiento divergente hacen relación a la habilidad de tomar diversas direcciones cuando uno se enfrenta a un problema.

Es pertinente resaltar la creatividad como aspectos inherente al proceso investigativo propiamente dicho y que orienta los procesos superiores de la actividad humana, en relación a la manera como cada individuo enfrenta y asume los diferentes espacios o responsabilidades con las que tiene que cumplir, trátese de un proyecto de investigación, los procesos de aprendizaje o de enseñanza que enmarca la educación superior o la resolución de problemas cotidianos.

Para ir complementando las ideas anteriores, se reconoce que los procesos de investigación son uno de los medios para generar conocimiento y acciones que posibiliten la solución de los problemas que aquejan a la humanidad.

La investigación también se puede abordar desde las dos perspectivas planteadas para la creatividad, es decir, como objeto de reflexión, con lo que se entra en el terreno de la epistemología, concibiéndola como aquella ciencia que indaga sobre los procesos de producción del conocimiento; si se aborda como metodología, se entiende que se enmarca en los parámetros del método científico que se orienta, como ya se dijo, hacia el esclarecimiento de la naturaleza de los fenómenos investigados, como es el caso de las investigaciones de base, y/o hacia la intervención en ellos, como es bien conocida la investigación aplicada.

Diferenciar estos dos aspectos es complicado ya que pensar el mismo proceso investigativo, la postura reflexiva - epistemológica, conlleva reflexionar sobre la metodología implementada por el investigador de manera reflexiva en torno a la naturaleza de su objeto de estudio y la forma de hacerlo tangible, observable, manipulable, valga la aclaración de que no se diferencian las dos posturas. Ya que se invita a una reflexión de orden epistemológico desde la manera como se acre- cienta el conocimiento científico y la mirada de la investigación cuando se le piensa desde el desarrollo de la creatividad y el logro de los objetivos de aprendizaje de la educación superior.

El desarrollo de competencias investigativas es uno de los objetivos de la educación superior y es objeto de procesos de enseñanza y aprendizaje que se establecen en cursos tendientes a generar saberes y competencias en esta área de trabajo y ellos, en términos generales, siguen una estructura básica centrada en el problema de investigación, en los objetivos, en el marco teórico, en la metodología, en la recolección y análisis de la información, como elementos básicos.

La competencia investigativa está orientada hacia el entendimiento de la investigación como una actividad que aglutina dimensiones como la epistemológica, metodológica, técnica y social. Este tipo de acción como investigación, la investigación, saberes, conocimientos, saber hacer en contexto investigativo con idoneidad y sentido ético,

Dentro de este proceso en la mayoría de las instituciones de educación superior se establece la realización de trabajos de grado investigativos como una opción para lograr el título profesional; la realización del mismo es un factor de ventaja comparativa, plus de capital intelectual para el ingreso a una maestría o doctorado, cuando se está pensando en continuar con la formación profesional.

La investigación a nivel del trabajo de grado se estructura sobre la premisa de que en la medida en que conozcamos la naturaleza de los fenómenos podremos intervenir en ellos para solucionarlos o aminorar el impacto negativo de los mismos en la sociedad, se ponen en ejecución varias tareas de orden práctico que nos llevan a entender que la investigación no solamente implica conocimientos o saberes sino que exige, sobre todo mucho hacer, mucha práctica; se concluye de estas premisas que "se aprende a investigar investigando". 
Entonces, desde esa intención de formación se establecen una serie de parámetros normativos que marcan la formación del estudiante dentro de ciertos postulados de rigor científico, éticos y morales; los que han de permitir afianzar y desarrollar la competencia investigativa.

Estas investigaciones, grosso modo, se clasifican, en cualitativas, cuantitativas o mixtas, dependiendo de la manera como se conciba el fenómeno y se opte por una perspectiva de abordaje; aquí queremos llamar la atención nuevamente sobre la creatividad puesta al servicio de un interés particular. Es decir, el investigador, apoyado en referentes contextual, teórico y metodológico, delimita el fenómeno y la manera de acercarse a él dentro del espectro científico que ofrecen los métodos.

La visión parcial de que los fenómenos sociales y humanos solo se pueden abordar a través de métodos cualitativos, o aquellos de las ciencias "exactas" a través de métodos cuantitativos es un poco restrictiva, limita y atenta contra la creatividad en investigación al establecer marcos estrechos y restrictivos que conciben el proceso investigativo como una lista de chequeo que constriñe y delimita las formas de acercamiento al objeto de estudio.

Por eso es importante tener presente que, identificar verdaderos problemas de investigación implica la demarcación del objeto de investigación dentro del campo disciplinar, esto es el “¿qué?", o sea, el contenido de la formación investigativa; si esto no está claro, no puede haber precisión en el “¿cómo?”, o sea, en los métodos de enseñanza aprendizaje necesarios para lograr dicha formación; siendo entonces, la relación entre contenido y método la que expresa la asunción de la investigación como un todo integral entre tema o contenido y proceso o actividad científico investigativa (Zuluaga Albarracín, 2016).

El investigador muestra su creatividad al tratar de establecer, fundamentar y explicar su manera de concebir el fenómeno de estudio, dentro de unos parámetros de coherencia y consistencia propios que determinan a los otros miembros de la comunidad académica ese objeto de estudio, es decir dentro de los parámetros del método científico.

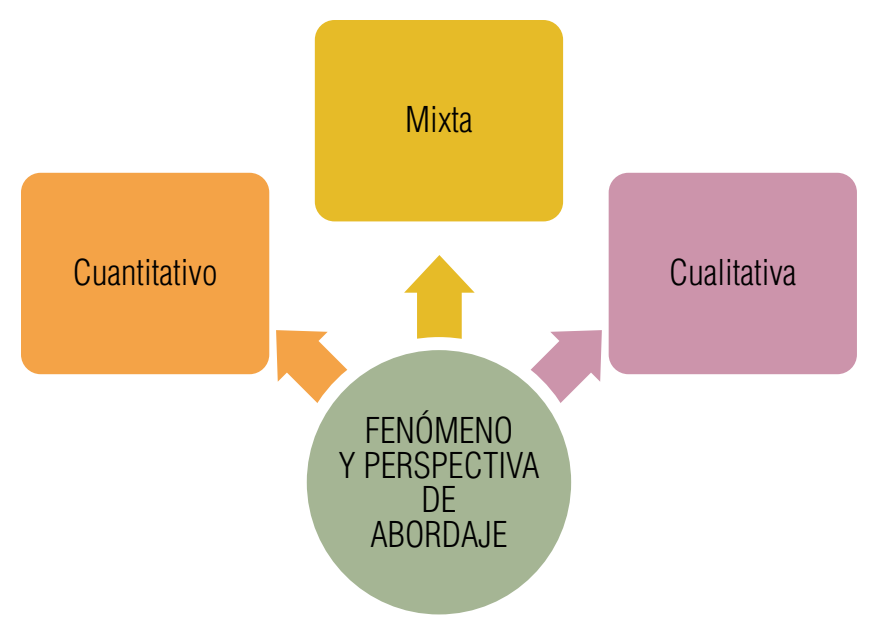

Gráfica 2. Tipos de investigación

Otra gran clasificación es la dada por el tipo de investigación, se reconocen las experimentales y no experimentales, la exploratoria, la descriptiva, la cuasi experimental, ex post facto, etc., entre otras y que se ajustan al nivel de reconocimiento del fenómeno y la manera como se establecen las variables intervinientes y las relaciones de causalidad $\mathrm{y}$, donde comúnmente, se señala la relación entre variables dependientes e independientes y el nivel de conocimiento de las mismas para recurrir a un modelo experimental o no.

En este contexto el énfasis lo realizamos en el diseño de investigación como el aparte que articula el acto investigativo, al integrar los aspectos relacionados con el problema, los objetivos de investigación y el marco teórico con la información recolectada, el procesamiento de la misma, su análisis y las conclusiones de la investigación.

La investigación es un escenario privilegiado para la construcción de conocimiento aplicado, un escenario para poner en funcionamiento las competencias del investigador, o del investigador en formación, para indagar en los fenómenos, concebirlos, caracterizarlos o para solucionar problemas. 
Se abordan a continuación las modalidades de trabajo de grado y lo que estas demandan de parte del estudiante en formación investigativa y de los escenarios educativos que pretenden desarrollar una serie de competencias vinculadas a lo investigativo. Estas se caracterizan por establecer con claridad los fenómenos en que se debe indagar a partir de métodos cualitativos, cuantitativos o mixtos.

La monografía. Requiere principalmente un análisis de documentos en torno a un fragmento de la realidad, la que se trata de comprender desde una perspectiva especial, la del investigador; éste trata de reflexionar en torno a una serie de datos escritos sometiéndolos a análisis documental, metodológicamente la información recolectada es organizada, estructurada y analizada para arrojar luz a la perspectiva del investigador, quien idealmente debe tener la objetividad suficiente y la flexibilidad para dejarse orientar por los datos.

Investigación Básica que puede ser de tipo experimental o no experimental teniendo en cuenta la manera como se conciban las variables involucradas y si el investigador puede y le interesa manipular en virtud de su investigación. Nótese que en todo momento se están marcando las bifurcaciones que encuentra el investigador y, por supuesto, sobre las opciones y problemas frente a los cuales el investigador debe asumir las decisiones más acertadas para lograr los objetivos propuestos.

Investigación Aplicada: Se fundamenta sobre la premisa de que es posible realizar una intervención que incida positivamente en un aspecto de la realidad estudiada, realidad que, una vez modificada, pueda ser objeto de análisis con la finalidad de medir el impacto de la intervención. Este tipo de investigación se estructura sobre la premisa de que se conoce el fenómeno sobre el que se pretende incidir (las variables intervinientes y como afectarlo), de tal manera que se ponen a prueba los supuestos de la investigación y se evalúa su nivel de incidencia. Este tipo de investigación se estructura sobre diseños de tipo experimental, realizando la graduación de los mismos en términos de pre-ex- perimentales - cuasi-experimentales. Es decir en ellos hay una manipulación de las variables intervinientes y un reconocimiento de que el fenómeno se puede transformar a partir de la acción del investigador, sea que se trate de una investigación cualitativa o cuantitativa.

La sistematización de una experiencia pedagógica o educativa, se deriva de un proyecto de intervención, el cual se ajusta a los parámetros de la investigación aplicada (en su intención de transformar la realidad), este tipo de trabajo de grado se analiza desde algunas de sus características, por lo es necesario entender qué es una sistematización.

En este sentido, en un primer momento se retoman los postulados de (Jara Holliday, 2001), en el documento titulado: "Dilemas y desafíos de la sistematización de experiencias", según el autor, existen dos acepciones para el concepto: la primera, concibe la sistematización como el ordenamiento y la clasificación de datos e informaciones de diversa índole, que se estructuran de tal manera que exige la elaboración de categorías, el establecimiento de relaciones, lo que permite, al final, la construcción de bases de datos organizados en cuerpos coherentes y cohesionados; la otra acepción, entendiendo la anfibología del concepto, se acerca mucho más a lo que se intenta demostrar y es que la sistematización tiene un sentido mucho más complejo, nos obliga a retomar la historia, ya que se trata de observar las experiencias como procesos diacrónicos $y$, por lo tanto, como secuencias de eventos encadenados; procesos en donde intervienen actores en contextos socio culturales e institucionales determinados por las variables espacio y tiempo.

Se puede ampliar el espectro de lo planteado por el autor y entender que una sistematización posee otras denominaciones que explicitan el sentido de su realización; entre otras definiciones, encontramos los postulados de Tréllez, quien argumenta que corresponde al culmen de un proceso de aprendizaje en donde elementos heteróclitos deben ser clasificados en órdenes jerárquicos, dice que la sistematización: 
Es el momento final de la secuencia y está dirigido a organizar los resultados del aprendizaje realizado a través de los momentos anteriores. Este proceso implica el desarrollo, entre otras destrezas, de la habilidad para establecer y manejar criterios de selección y ordenamiento; de la habilidad para discernir entre lo importante y trascendente y lo que es superfluo o circunstancial y de la capacidad de establecer categorías y jerarquías de valoración (Tréllez Solís, 2010).

Esta última denominación puede ser ampliada en la perspectiva de la concepción de la pedagoga Cendales González (2003), que señala que ésta corresponde a un tipo de investigación cualitativa reconocida como investigación socio crítica y de carácter interpretativo. Este tipo de investigación está orientada hacia la comprensión y la interpretación de las experiencias por parte de los actores o protagonistas; aquí se los interroga para saber sobre sus percepciones acerca de los fenómenos y sus lógicas de ordenamiento de los saberes y del propio proceso llevado a cabo; además de lograr identificar los intereses que se ponen en juego y, en últimas, para profundizar en las propias representaciones de los involucrados en la experiencia.

La misma investigadora dice que en el trasfondo de cada sistematización hay un concepto de realidad que puede ser comprendida como el contexto del sujeto, como lo externo a él, o como el producto de la construcción colectiva en la intersubjetividad, es decir, desde la interacción social en el ámbito de la cultura. Concluye, que esa realidad está cruzada por una serie de múltiples elementos de tipo contextual, subjetivo o racional y que dichos elementos constituyen el objeto de la sistematización (Cendales González, 2003)

La definición que nos ofrece Sonia Lavín, por otro lado, se estructura desde un foco central que busca comprender el sentido escondido de la experiencia, porque ella no se presenta plena en su totalidad, este sentido se oculta en algún lugar y sus elementos constitutivos están diluidos o diseminados en partes desiguales dentro de la ingente cantidad de factores que son susceptibles de ser tomados en cuenta como materia prima bruta de la sistemati- zación; la investigadora, entonces, plantea que la sistematización es:

Un proceso de construcción de conocimiento. La sistematización se hace para producir conocimiento útil. Quien hace sistematización piensa que se puede aprender de la práctica y que ese conocimiento obtenido ayudará a mejorar esa práctica. Nos planteamos sistematizar especialmente cuando entendemos que en una determinada experiencia o proceso hay una riqueza oculta o no exprimida en términos de conocimiento útil para el futuro y que la cantidad y calidad de ese conocimiento esperado nos hacen prever como deseable o rentable la sistematización (desde todos los puntos de vista, no solo el económico) (Lavín Herrera, 2010).

Llegados a este punto de la reflexión, podemos ir decantando esta aproximación conceptual desde nuestra propia experiencia como docentes que dirigen de trabajos de grado en programas de educación superior; el objetivo principal de dichos trabajos consiste en sistematizar experiencias pedagógicas o educativas.

Partiendo de dichas consideraciones, la sistematización es una extensa indagación sobre la multiplicidad de factores que intervienen en los procesos históricos; una pregunta inicial, que no se puede soslayar y que le imprime a la sistematización una contundente importancia es la pregunta por el sentido de los sucesos que intentamos comprender e interpretar, es decir, por su significado profundo dentro de las experiencias humanas. Dicha pregunta se desglosa en el intento que realiza la sistematización ordenando y reconstruyendo lo que ha sucedido en el proceso (Jara Holliday, 2001).

La sistematización es entonces una reconstrucción de experiencias desde lo sucedido en un pasado tanto alejado como reciente, el ejercicio se inicia desde los distintos elementos de orden subjetivo o de orden objetivo para comprender, interpretar y aprender de nuestra propia práctica. 
Se introduce de forma subrepticia el concepto de educación bajo el ropaje del concepto aprendizaje; el aprender de nuestra propia experiencia no deja de lado dos elementos de la tríada de la educación como son el enseñar y el aprender. Llegamos a entender la sistematización como una mirada crítica sobre el significado de los procesos disertados, de tal manera que sus piezas desarticuladas y, nuevamente, articuladas en la acción interpretativa y comprensiva, nos permitan extraer aprendizajes que puedan ser útiles en un futuro.

El aprendizaje como experiencia posibilita mejorar nuestra propia práctica en muchos sentidos, no se trata solamente de que los pasos se delimiten por la reconstrucción y el ordenamiento de la información; se trata de que las fases del proceso de sistematización se vislumbren desde una perspectiva de interpretación crítica, para fomentar así el acervo sobre las teorías sociales y culturales y también para compartir los aprendizajes con experiencias comunes que se retroalimenten entre sí desde la similaridad de los elementos constituyentes.

El tipo de experiencia a sistematizar constriñe y, a la vez, orienta la metodología que se emplea en el ejercicio, en nuestro caso, se trataba de sistematizar una experiencia pedagógica, cuya metodología se comprende como una práctica de intervención con miras a solucionar un problema detectado en el aula de clases. Dicho panorama plantea, sobre todo, una investigación diagnóstica en la fase inicial que identifique un problema.

El componente histórico de una sistematización incluye responder a los interrogantes relacionados con ¿Qué pasó? ¿Cómo pasó? ¿Quiénes intervinieron y participaron de las fases del proceso? ¿Qué relaciones se establecieron? ¿Qué de la experiencia es pertinente replicar? ¿Cómo mi experiencia y saber es útil en otros contextos?

La sistematización de experiencias pedagógicas evidencia que la investigación es un proceso activo y creativo tendiente a indagar en un aspecto de la realidad educativa, la mirada del investigador permite conceptualizar, concebir y operar sobre ese fenómeno desde un proceso investigativo; posibilita, entonces, un ordenamiento de los conocimientos útiles para otros contextos en donde se presenten problemáticas semejantes. De esta manera el inventario de conocimientos permite nutrir el acervo conceptual, teórico y práctico de esa parcela del saber, en nuestro del inmenso campo del saber pedagógico.

\section{CONCLUSIONES}

La investigación permite desarrollar competencias profesionales e investigativas relacionadas con el saber ser, saber hacer y saber pensar en contexto y aquí se puede concluir cómo la formación de la educación superior encuentra su sentido misional de la proyección en el ansiado diálogo con la sociedad.

La sistematización de experiencias pedagógicas, por último, posibilita que se logren los objetivos de la educación superior formando personas que puedan interrogar la realidad desde perspectivas críticas, desde nuevas miradas que arrojen nuevas luces e ideas y que fomenten respuestas innovadoras ante las múltiples problemáticas que se encuentran en el universo educativo.

La sistematización de experiencias pedagógicas como elemento de formación educativa propicia la formación de competencias de investigación que legitiman un ejercicio de indagación y reflexión sobre problemáticas educativas. Lleva al estudiante a pensar de manera práctica, es decir, en el contexto, sobre la riqueza y utilidad de los paradigmas de investigación y de su área de formación, para tratar de objetivar elementos de la realidad y preguntarse sobre las variables que intervienen en él; ejercicio que llevado de la mano del rigor científico, le da elementos para pensar de forma clara, objetiva, coherente y viable; con lo que se desarrollan competencias que trascienden el ámbito investigativo y que son útiles en el que- 
hacer del profesional que día a día enfrenta situaciones que desbordan lo enseñado o lo aprendido en términos de saber acumulado.

\section{BIBLIOGRAFÍA}

(s.f.).

Cendales González, L. (2003). La sistematización de experiencias. organizado por el Centro Latinoamericano y del Caribe para el Desarrollo Cultural (CLACDEC) del Consejo Nacional de la Cultura (CONAC). Recuperado el 27 de Septiembre de 2012, de http://www.mimdes.gob.pe/files/ DIRECCIONES/DGPDS/sistematizacion_marco.pdf

Huidobro Salas, T., \& Gonzáles Marquéz, J. (2005). Una definición de la creatividad a través de 24 autores seleccionados. España: Universidad Complutense de Madrid. Obtenido de http://site.ebrary.com/lib/ uniajccsp/Doc?id=10091127\&ppg=129

Jara Holliday, Ó. (Abril de 2001). Dilemas y desafíos de la sistematización de experiencias. Cochabamba, Bolivia: Intercooperation, s.p.

Lavín Herrera, S. (2010). Marco conceptual de la sistematización de experiencias. Lima, Peru. Recuperado el 27 de Septiembre de 2012, de http://www.mimdes. gob.pe/files/DIRECCIONES/DGPDS/sistematizacion_marco.pdf

Manrique Mosquera, J. C., \& Zuluaga Albaracín, H. (Junio - Julio de 2015). Abordaje Conceptual y Metodologico de los Imaginarios de las Matematicas. Educação Matemática em Revista, 2 (01), 13-25. Recuperado el 16 de Marzo de 2019, de http://www. famper.com.br/arquivos/revistaeletronica/abordaje-conceptual-y-metodologico-de-los-imaginarios-de-las-matematicas_1432585370.pdf

Tréllez Solís, E. (2010). Marco conceptual de la sistematización de experiencias. Lima, Peru: Dirección General de Políticas de Desarrollo Social Dirección de
Investigación y Desarrollo Social, Li. Recuperado el 27 de Septiembre de 2012, de http://www.mimdes. gob.pe/files/DIRECCIONES/DGPDS/sistemati

\section{LOS AUTORES}

Hernán Zuluaga Albarracín: Antropólogo, Universidad del Cauca; Especialista en Investigación Educativa Universitaria, USB CINDE; Aspirante al Doctorado en Ciencias Pedagógicas, Universidad Enrique José Varona, Habana - Cuba; Docente tiempo completo Institución Universitaria Antonio José Camacho. Miembro del Grupo de Investigación en Pedagogía, GIP.hzalbarracin@gmail.com

Juan Carlos Manrique Mosquera: Psicólogo, Universidad del Valle; Mg. en Psicología con énfasis en Desarrollo Cognitivo, Universidad del Valle; Aspirante al Doctorado en Ciencias Pedagógicas, Universidad Enrique José Varona, Habana - Cuba; Docente tiempo completo Institución Universitaria Antonio José Camacho. Miembro del Grupo de Investigación en Pedagogía, GIP. Lider del semillero de investigación Siembra - Uniajc. jmanrique@hotmail.com 